\title{
Some remarks on adaptive stabilization of infinite-dimensional systems
}

\author{
Hartmut Logemann \\ Institute for Dynamical Systems, University of Bremen, \\ Postfach 330440, 2800 Bremen 33, FRG
}

\section{Hans Zwart \\ Department of Applied Mathematics, University of Twente, P.O. Box 217, 7500 AE Enschede, Netherlands}

Received 18 August 1990

Abstract: It is the purpose of this note to show that a first-order adaptive controller stabilizes a large class of infinite-dimensional systems described by strongly continuous semigroups. It is assumed that the plant is minimum-phase and has invertible high-frequency gain. Knowledge of the sign of the highfrequency gain is not required.

Keywords: Infinite-dimensional systems; adaptive stabilization; strongly continuous semigroups; semilinear evolution equations.

\section{Introduction}

Generalizing a result by Nussbaum [12] Willems and Byrnes [15] constructed a sign-switching high-gain adaptive controller which globally stabilizes any finite-dimensional single-input single-output minimum-phase system with invertible high-frequency gain. In recent years it was shown by several authors (see Dahleh [3], Dahleh and Hopkins [4], Kobayashi [7], Logemann [8] and Logemann and Owens [9]) that the adaptive algorithm presented in [15] stabilizes certain classes of infinite-dimensional systems as well. In [3], [4] and [8] the main result of [15] was extended to various classes of retarded systems. Generalizations to distributed parameter systems described by analytic semigroups were given in [7], while an input-output theory of high-gain adaptive stabilization of systems described by non-rational transfer functions was developed in [9].
In the following we shall consider systems of the form

$\dot{x}=A x+B u, \quad y=C x$,

where $A$ generates a strongly continuous semigroup $S(t)$ on a Banach space $X$ and $B: \mathbb{R} \rightarrow X$ and $C: X \rightarrow \mathbb{R}$ are bounded linear operators. Suppose that the system (1.1) has no zeros in $\operatorname{Re}(s) \geq \alpha$ for some $\alpha<0$ and $C B \neq 0$. Under these conditions it was shown by Kobayashi [7] that the adaptive control law given in [15] will globally stabilize the system (1.1) provided that

(i) $X$ is a Hilbert space,

(ii) $A$ is selfadjoint and has a complete orthonormal system of eigenvectors,

(iii) $S(t)$ is analytic,

(iv) im $B$ and im $C^{*}$ are contained in the domain of $A$.

In this paper we will answer the question posed in [7] whether the conditions (iii) and (iv) are really necessary for adaptive stabilization. We will show that (i)-(iv) can be relaxed considerably. In particular it will turn out that

- (i)-(iii) can be dropped,

- (iv) can be relaxed if (iii) holds.

The paper is organized as follows. Section 2 is devoted to preliminaries concerning the class of systems under consideration. Moreover it contains some technical lemmas which will be used in Section 3 in order to prove the main results of this paper. In the Appendix we prove the existence of a well-defined transfer function for a class of infinite-dimensional systems with unbounded observation operator. This result, which is needed in Section 3, might be of some independent interest.

\section{Notation}

For $\alpha \in \mathbb{R}$ define

$\mathbb{C}_{\alpha}:=\{s \in \mathbb{C} \mid \operatorname{Re}(s)>\alpha\}$. 
Let $H_{\alpha}^{\infty}$ denote the algebra of functions which are analytic and bounded on $\mathbb{C}_{\alpha}$.

Let $X$ and $Y$ be normed spaces. The vector space of all linear bounded operators from $X$ to $Y$ is denoted by $\mathscr{L}(\mathrm{X}, \mathrm{Y})$.

Let $A$ be a linear operator. Then we define $D(A):=$ domain of $A, \sigma(A):=$ spectrum of $A$ and $\rho(A):=$ resolvent set of $A$.

\section{Preliminaries and system description}

In the following we shall consider systems of the form

$$
\begin{aligned}
& \dot{x}(t)=A x(t)+B u(t), \quad x(0)=x_{0}, \\
& y(t)=C x(t), \quad t \geq 0,
\end{aligned}
$$

where $A$ generates a strongly continuous semigroup $S(t)$ on a real Banach space $X, B \in$ $\mathscr{L}(\mathbb{R}, X)$ and $C \in \mathscr{L}(X, \mathbb{R})$. Sometimes it will be necessary to consider the complexifications of $X$, $A, B$, and $C$. For simplicity these will be denoted by $X, A, B$, and $C$ as well.

The notion of exponential stabilizability will play an important role in the sequel.

2.1. Definition. The system (2.1) (or the pair $(A, B)$ ) is called exponentially stabilizable if there exists $K \in \mathscr{L}(X, \mathbb{R})$ such that the strongly continuous semigroup generated by $A+B K$ is exponentially stable.

2.2. Lemma. Suppose that the pair $(A, B)$ is exponentially stabilizable and $\sigma(A) \subset \mathbb{C} \backslash \mathbb{C}_{\alpha}$ for some $\alpha<0$. Then the strongly continuous semigroup $S(t)$ generated by $A$ will be exponentially stable.

The proof of the above lemma follows easily from Nefedov and Sholokhovich [11] or Jacobson and Nett [5] (cf. also Curtain [2]).

The following definition will make precise what we mean by a zero of the system (2.1).

2.3. Definition. A number $\lambda \in \mathbb{R}$ is called a zero of the system (2.1) if the kernel of the operator

$\left(\begin{array}{cc}\lambda I-A & B \\ C & 0\end{array}\right): D(A) \oplus \mathbb{C} \rightarrow X \oplus \mathbb{C}$

is non-trivial.
2.4. Remark. Let $\lambda \in \mathbb{C}$ be a zero of the system (2.1) and suppose that $\lambda \in \rho(A)$. Then it is easy to show that $\lambda$ is a zero of the transfer function

$G(s)=C(s I-A){ }^{11} B$

of (2.1).

Let us introduce the following assumptions (A1) $C B \neq 0$.

(A2) The system (2.1) has no zeros in $\mathbb{C}_{a}$ for some $\alpha<0$.

(A3) The system (2.1) is exponentially stabilizable.

(A4) im $B \subset D(A)$.

(A5) im $C^{*} \subset D\left(A^{*}\right)$.

(A6) im $B \subset D\left(A^{2}\right)$.

(A7) im $C^{*} \subset D\left(A^{* 2}\right)$.

The next lemma establishes the existence of a feedback operator which shifts the spectrum of $A$ into the left half plane.

2.5. Lemma. Let (A1)-(A3) be satisfied and define

$F_{\gamma}:=(C B)^{-1}(-C A+\gamma C)$,

where $\gamma<0$. Then there exists $\alpha \in(\gamma, 0)$ such that $\sigma\left(A+B F_{\gamma}\right) \subset \mathbb{C} \backslash \mathbb{C}_{\alpha}$.

Proof. By (A3) there exists $\beta<0$ such that the spectrum of $A$ in $\mathbb{C}_{\beta}$ consists of isolated eigenvalues with finite multiplicities (see Jacobson and Nett [5] or Curtain [2]). Moreover we have

$\overline{\mathrm{C}}_{0} \cap \rho\left(A+B F_{\gamma}\right) \neq \varnothing$

by Appendix I. Since $B F_{Y}$ is an $A$-degenerate operator it follows from Theorem 6.2 and Theorem 6.5 in Chapter IV of Kato's book [6] that the spectrum of $A+B F_{\gamma}$ in $\mathbb{C}_{\beta}$ consists of at most countably many eigenvalues with finite multiplicities. By (A2) there exists a number $\alpha<0$ such that the system (2.1) has no zeros in $\mathbb{C}_{\alpha}$. W.l.o.g. we may assume $\max (\beta, \gamma)<\alpha$. Suppose that there exists $\lambda$ in $\sigma\left(A+B F_{\gamma}\right) \cap \mathbb{C}_{\alpha}$. Then $\lambda$ is an eigenvalue of $A+B F_{y}$ and there exists $x \in X, x \neq 0$ such that

$\left(\lambda I-A-B F_{\gamma}\right) x=0$.

Hence

$\left(\lambda I-A+B(C B)^{-1} C A\right) x-\gamma B(C B)^{-1} C x=0$. 
Applying $C$ to both sides of the above equation gives $(\lambda-\gamma) C x=0$. Since $\gamma<\alpha<\operatorname{Re}(\lambda)$ it follows that $C x=0$. We obtain using (2.3),

$\left(\begin{array}{cc}\lambda I-A & B \\ C & 0\end{array}\right)\left(\begin{array}{c}x \\ (C B)^{-1} C A x\end{array}\right)=\left(\begin{array}{l}0 \\ 0\end{array}\right)$.

Thus $\lambda$ is a zero of (2.1) which is not possible by assumption. Hence we have shown

$\sigma\left(A+B F_{\gamma}\right) \cap \mathbb{C}_{\alpha}=\emptyset$.

2.6. Lemma. Let $\mathrm{F}_{\gamma}$ be defined as in (2.2). Suppose that (A1) holds and that $A+B F_{\gamma}$ generates $a$ strongly continuous semigroup $S_{\gamma}(t)$. Under these conditions we have

$S_{\gamma}(t) \operatorname{ker} C \subset \operatorname{ker} C \quad \forall t \geq 0$.

Proof. Let $x \in \operatorname{ker} C$. For $s \in \rho\left(A+B F_{\gamma}\right)$ we have

$$
\begin{aligned}
x= & \left(s I-A-B F_{\gamma}\right)\left(s I-A-B F_{\gamma}\right)^{-1} x \\
= & {\left[s I-A-B(C B)^{-1}(-C A+\gamma C)\right] } \\
& \cdot\left(s I-A-B F_{\gamma}\right)^{-1} x .
\end{aligned}
$$

Applying $C$ to both sides of the above equation we obtain

$$
\begin{aligned}
0 & =(s C-C A+C A-\gamma C)\left(s I-A-B F_{\gamma}\right)^{-1} x \\
& =(s-\gamma) C\left(s I-A-B F_{\gamma}\right)^{-1} x .
\end{aligned}
$$

Hence we have shown for all $s \in \rho\left(A+B F_{\gamma}\right)$, $s \neq \gamma$, that

$\left(s I-A-B F_{\gamma}\right)^{-1}$ ker $C \subset \operatorname{ker} C$.

The claim now follows from Pazy [13], p. 121.

2.7. Remark. The feedback law $F_{\gamma}$ was introduced by Curtain in [1], Section 8 in the context of disturbance decoupling for infinite-dimensional systems (cf. also Zwart [17]).

\section{Main results}

Let us recall the definition of a Nussbaum gain.

3.1. Definition. A measurable locally bounded function $N: \mathbb{R} \rightarrow \mathbb{R}$ is called a Nussbaum gain if for some $t_{0} \in \mathbb{R}$,

$$
\sup _{t>t_{0}} \frac{1}{t-t_{0}} \int_{t_{0}}^{t} \tau N(\tau) \mathrm{d} \tau=+\infty
$$

and

$\inf _{t>t_{0}} \frac{1}{t-t_{0}} \int_{t_{0}}^{t} \tau N(\tau) \mathrm{d} \tau=-\infty$.

3.2. Example. A continuously differentiable Nussbaum gain is given by

$N(\tau)=\cos \left(\frac{1}{2} \pi \tau\right) \exp \left(\tau^{2}\right)$,

cf. Nussbaum [12] or Logemann and Owens [9].

In this section we shall apply the following control law to the system (2.1):

$u(t)=N(k(t)) k(t) y(t)$,

$\dot{k}(t)=y^{2}(t), \quad k(0)=k_{0} \in \mathbb{R}$,

where $N$ is a Nussbaum gain. The control law (3.1) has been introduced by Willems and Byrnes [15] for finite-dimensional systems.

Defining

$A_{\mathrm{c}}: D(A) \times \mathbb{R} \rightarrow X \times \mathbb{R}$,

$\left(\begin{array}{l}x \\ k\end{array}\right) \mapsto\left(\begin{array}{c}A x \\ 0\end{array}\right)$

$f: X \times \mathbb{R} \rightarrow X \times \mathbb{R}$,

$\left(\begin{array}{l}x \\ k\end{array}\right) \mapsto\left(\begin{array}{c}N(k) k B C x \\ (C x)^{2}\end{array}\right)$,

and

$x_{\mathrm{c}}(t):=\left(\begin{array}{l}x(t) \\ k(t)\end{array}\right)$

we can write the closed-loop system as follows:

$\dot{x}_{\mathrm{c}}(t)=A_{\mathrm{c}} x_{\mathrm{c}}(t)+f\left(x_{\mathrm{c}}(t)\right), \quad t \geq 0$,

$x_{\mathrm{c}}(0)=\left(\begin{array}{l}x_{0} \\ k_{0}\end{array}\right) \in X \times \mathbb{R}$.

A continuously differentiable $D\left(A_{\mathrm{c}}\right)$-valued function which satisfies (3.2) is called a classical solution of (3.2). A mild solution of (3.2) is a continuous function satisfying

$x_{\mathrm{c}}(t)=S_{\mathrm{c}}(t) x_{\mathrm{c}}(0)+\int_{0}^{t} S_{\mathrm{c}}(t-\tau) f\left(x_{\mathrm{c}}(\tau)\right) \mathrm{d} \tau$,

where $S_{\mathrm{c}}(t)$ denotes the strongly continuous semigroup generated by $A_{\mathrm{c}}$.

The following lemma shows that (3.2) is wellposed. 
3.3. Lemma. (i) If $N$ satisfies a local Lipschitz condition then for all $x_{\mathrm{c}}(0) \in X \times \mathbb{R}$, (3.2) has a unique mild solution which can be continued to the right as long as it remains bounded.

(ii) If $N$ is continuously differentiable then for all $x_{\mathrm{c}}(0) \in D(A) \times \mathbb{R},(3.2)$ has a unique classical solution which can be continued to the right as long as it remains bounded.

Proof. (i) It is easy to show that $f$ satisfies a local Lipschitz condition, i.e. for any $l>0$ there exists $L>0$ such that

$$
\left\|f(z)-f\left(z^{\prime}\right)\right\| \leq L\left\|z-z^{\prime}\right\|
$$

for all $z, z^{\prime} \in X \times \mathbb{R}$ satisfying $\|z\|,\left\|z^{\prime}\right\| \leq l$, where the norm $\|\cdot\|$ on $X \times \mathbb{R}$ is defined by $\|\cdot\|=\|\cdot\|_{X}+|\cdot|$. The claim follows now from Segal [14], Theorem 1 (cf. also Pazy [13], pp. 185).

(ii) It is routine to show that $f$ is continuously Fréchet-differentiable. Moreover $f$ satisfies a local Lipschitz condition (notice that this does not follow necessarily from the $C^{1}$-property in the infinite-dimensional case). Application of Theorem 1 and Lemma 3.1 in Segal [14] (cf. also Martin [10], pp. 347) proves the claim.

We are now in the position to state our main results.

3.4. Theorem. Suppose that assumptions (A1)-(A5) are satisfied and that $N$ is a continuously differentiable Nussbaum gain. The following statements hold true.

(i) For all $\left(x_{0}, k_{0}\right) \in D(A) \times \mathbb{R}$ the closed-loop system given by (2.1) and (3.1) has a unique globally defined classical solution $(x(t), k(t))$ with the following properties:

$\lim _{t \rightarrow \infty} k(t)$ exists and is finite,

$x(\cdot) \in L^{2}(0, \infty ; X) \cap L^{\infty}(0, \infty ; X)$,

$\lim _{t \rightarrow \infty} x(t)=0$.

(ii) For all $\left(x_{0}, k_{0}\right) \in X \times \mathbb{R}$ the closed-loop system given by (2.1) and (3.1) has a unique globally defined mild solution $(x(t), k(t))$ satisfying (3.3)-(3.5).

Proof. (i) Define the linear bounded operator $P_{1}: X \rightarrow X$ by

$P_{1} x=B(C B)^{-1} C x$.
Then $P_{1}$ is a projection and $\operatorname{im} P_{1}=\operatorname{im} B$. Moreover set $P_{2}:=I-P_{1}$. It is obvious that im $P_{2}=$ ker $C$ and $X=\operatorname{im} B \oplus \operatorname{ker} C$. Let $(x(t), k(t))$ denote the classical solution of the feedback system given by (2.1) and (3.1) with initial value $\left(x_{0}, k_{0}\right)$ $\in D(A) \times \mathbb{R}$ and let $\left[0, t_{0}\right)$ denote its maximal interval of existence. Realizing that

$\left(A+B F_{Y}\right)(D(A) \cap \operatorname{ker} C) \subset \operatorname{ker} C$,

im $P_{1} \subset D(A), \quad P_{2}(D(A)) \subset D(A)$

and

$P_{1} A P_{2} x=-B F_{\gamma} P_{2} x \quad \forall x \in D(A)$,

we obtain from (2.1),

$$
\begin{aligned}
P_{1} \dot{x}(t) & =P_{1} A x(t)+B u(t) \\
& =P_{1} A P_{1} x(t)-B F_{\gamma} P_{2} x(t)+B u(t)
\end{aligned}
$$

and

$$
\begin{aligned}
P_{2} \dot{x}(t) & =P_{2}\left(A+B F_{\gamma}\right) x(t) \\
& =\left(A+B F_{\gamma}\right) P_{2} x(t)+P_{2} A P_{1} x(t) .
\end{aligned}
$$

Noticing that $P_{1} x(t)=B(C B)^{-1} y(t)$ and setting $z(t):=P_{2} x(t)$ it follows

$$
\begin{gathered}
B(C B)^{-1} \dot{y}(t)=B(C B)^{-1} C A B(C B)^{-1} y(t) \\
+B\left(u(t)-F_{\gamma} z(t)\right), \\
\dot{z}(t)=\left(A+B F_{\gamma}\right) z(t)+P_{2} A B(C B)^{-1} y(t) .
\end{gathered}
$$

We conclude that the initial value problem given by (2.1) and (3.1) can be written as

$$
\begin{aligned}
\dot{y}(t)= & C B v_{1}(t), \quad y(0)=C x_{0}, \\
\dot{z}(t)= & \left(A+B F_{\gamma}\right) z(t) \\
& +P_{2} A B(C B)^{-1} v_{2}(t), \quad z(0)=P_{2} x_{0}, \\
w(t)= & F_{\gamma} z(t)-(C B)^{-1} C A B(C B)^{-1} v_{2}(t), \\
v_{1}(t)= & u(t)-w(t), \quad v_{2}(t)=y(t), \\
\dot{k}(t)= & y^{2}(t), \quad k(0)=k_{0}, \\
u(t)= & N(k(t)) k(t) y(t) .
\end{aligned}
$$

Hence we have shown that $(x(t), k(t))$ solves the initial value problem given by (2.1) and (3.1) (where $x_{0} \in D(A)$ ) on $\left[0, t_{0}\right.$ ) if and only if

$x(t)=z(t)+B(C B)^{-1} y(t)$, 
where $(z(t), y(t), k(t))$ is a solution of the initial value problem defined by (3.6)-(3.9) on $\left[0, t_{0}\right)$.

We obtain from (A4) and (A5) that $P_{2} A B$ $(C B)^{-1}, F_{\gamma}$ and $(C B)^{-1} C A B(C B)^{-1}$ ) are bounded linear operators. Hence it follows in particular that $A+B F_{\gamma}$ generates a strongly continuous semigroup which will be denoted by $S_{\gamma}(t)$. Using Lemma 2.6 we obtain that $S_{\gamma}(t)$ is a strongly continuous semigroup on $\operatorname{ker} C$. Therefore (3.7) is a well-defined semigroup system on $\operatorname{ker} C$. Clearly, by (A3), the pair $\left(A+B F_{\gamma}, B\right)$ is exponentially stabilizable. Applying Lemma 2.2 and Lemma 2.5 we see that $S_{y}(t)$ is exponentially stable. As a consequence the transfer function of (3.7) is in $H_{\alpha}^{\infty}$ for some $\alpha<0$. It now follows from Logemann and Owens [9] that the pair $(y(t), k(t))$ is bounded on $\left[0, t_{0}\right)$ which implies via (3.7) and (3.10) that $(x(t), k(t))$ is bounded on $\left[0, t_{0}\right)$. Using Lemma 3.3(ii) we obtain $t_{0}=\infty$, i.e. the closed-loop system given by (2.1) and (3.1) has a unique globally defined classical solution. Finally it follows again from Logemann and Owens [9] that (3.3)-(3.5) hold with $x$ replaced by $y$, which proves the claim because of (3.10) and the exponential stability of (3.7).

(ii) It follows as in the proof of (i) that $(y(t), k(t))$ is bounded on $\left[0, t_{0}\right)$. Hence, by the exponential stability of (3.7) and Lemma 3.3(i) we have that the mild solution $(z(t), y(t), k(t))$ of the initial value problem (3.6)-(3.9) is globally defined. Moreover as in the proof of (i) we conclude that (3.3)-(3.5) hold true with $x$ replaced by $y$. In order to prove the claim it is sufficient to show that

$$
\left(z(t)+B(C B)^{-1} y(t), k(t)\right)
$$

is the mild solution of the initial value problem given by (2.1) and (3.1). We have already shown in the proof of (i) that this is true if $x_{0} \in D(A)$. Therefore it remains true in the general case (i.e. $\left.x_{0} \in X\right)$, since $D(A)$ is dense in $X$ and mild solutions depend continuously on their initial values (cf. Segal [14], Corollary 1.5).

3.5. Remark. (i) Notice that in the proof of Theorem 3.4 we have decomposed the original plant (2.1) into a feedback system consisting of an integrator in the forward loop and an (exponentially) stable system in the feedback loop (see (3.6)-(3.8)). Adaptive stabilization of systems ad- mitting such a decomposition has been investigated by Logemann and Owens [9] using an input-output approach.

(ii) Kobayashi [7] proved a result similar to Theorem 3.4. However he had to assume that $X$ is a Hilbert space and that $A$ is a selfadjoint operator on $X$ having complete orthonormal system of eigenvectors and generating an analytic semigroup. In particular Theorem 3.4 gives an affirmative answer to the question posed in [7] whether the assumption on the analyticity of the semigroup can be relaxed.

3.6. Corollary. Suppose that assumptions (A1)-(A3) and (A6) are satisfied and that $N$ is a continuously differentiable Nussbaum gain. Under these conditions statement (i) of Theorem 3.4 holds true.

Proof. Let $\lambda \in \rho(A)$ and define a new state-space system $(\tilde{A}, \tilde{B}, \tilde{C})$ by $\tilde{A}:=A, \tilde{B}:=(\lambda I-A) B$ and $\tilde{C}:=C(\lambda I-A)^{-1}$. Notice that the transfer functions of $(A, B, C)$ and $(\tilde{A}, \tilde{B}, \tilde{C})$ are the same. It is clear that $\tilde{A}, \tilde{B}$ and $\tilde{C}$ satisfy (A1), (A2), (A4) and (A5). Moreover it follows from Jacobson and Nett [5] or Curtain [2] via (A3) that $(\tilde{A}, \tilde{B})$ is exponentially stabilizable. Let $x_{0} \in D(A)$ and denote the mild solution of

$$
\begin{aligned}
& \dot{x}(t)=\tilde{A} x(t)+\tilde{B} u(t), \quad x(0)=(\lambda I-A) x_{0}, \\
& y(t)=\tilde{C} x(t), \\
& u(t)=N(k(t)) k(t) y(t), \\
& \dot{k}(t)=y^{2}(t), \quad k(0)=k_{0},
\end{aligned}
$$

by $(\tilde{x}(t), \tilde{k}(t))$. It follows from Theorem 3.4(ii) that $(\tilde{x}(t), \tilde{k}(t))$ is globally defined and satisfies (3.3)-(3.5) with $x$ and $k$ replaced by $\tilde{x}$ and $\tilde{k}$. Finally notice that the pair $(x(t), k(t))$ defined by

$$
x(t):=(\lambda I-A)^{-1} \tilde{x}(t) \text { and } k(t):=\tilde{k}(t)
$$

is a classical solution of the initial value problem given by (2.1) and (3.1).

3.7. Corollary. Suppose that the assumptions (A1)(A3) and (A7) are satisfied and that $N$ is a continuously differentiable Nussbaum gain. Under these conditions the statements (i) and (ii) of Theorem 3.4 hold true.

Proof. Let $\lambda \in \rho(A)$ and define $\tilde{A}:=A, \tilde{B}:=(\lambda I$ $-A)^{-1} B$ and $\tilde{C}:=C(\lambda I-A)$. As in the proof of 
Corollary 3.6 we have that the system given by $(\tilde{A}, \tilde{B}, \tilde{C})$ satisfies (A1)-(A5). Let $x_{0} \in X$ and denote the classical solution of

$\dot{x}(t)=\tilde{A} x(t)+\tilde{B} u(t), \quad x(0)=(\lambda I-A)^{-1} x_{0}$,

$y(t)=\tilde{C} x(t)$,

$u(t)=N(k(t)) k(t) y(t)$,

$\dot{k}(t)=y^{2}(t), \quad k(0)=k_{0}$,

by $(\tilde{x}(t), \tilde{k}(t))$. By Theorem 3.4(i), $(\tilde{x}(t), \tilde{k}(t))$ is globally defined and satisfies (3.3)-(3.5) with $x$ and $k$ replaced by $\tilde{x}$ and $\tilde{k}$. Notice that the pair $(x(t), k(t))$ defined by

$x(t):=(\lambda I-A) \tilde{x}(t)$ and $k(t):=\tilde{k}(t)$

is a mild solution of the initial value problem given by (2.1) and (3.1). It will be a solution in the classical sense if $x_{0} \in D(A)$. We have

$\tilde{x}(t)=S(t) \tilde{x}_{0}+\int_{0}^{t} S(t-\tau) \tilde{B} \tilde{u}(\tau) \mathrm{d} \tau$,

where $\tilde{x}_{0}:=(\lambda I-A)^{-1} x_{0}$ and

$\tilde{u}(t):=N(\tilde{k}(t)) \tilde{k}(t) C \tilde{x}(t)$.

Since the pair $(\tilde{A}, \tilde{B})$ is exponentially stabilizable, there exist closed subspaces $X_{s}$ and $X_{u}$ of $X$ such that

- $X=X_{s} \oplus X_{u}, \quad X_{u}$ is finite-dimensional and $X_{u} \subset D(\tilde{A})=D(A)$

- the projections $P_{s}: X \rightarrow X_{s}$ and $P_{u}: X \rightarrow X_{u}$ commute with $S(t)$ and $\tilde{A}=A$;

- the strongly continuous semigroup $S_{s}(t):=$ $\left.S(t)\right|_{X_{s}}$ on $X_{s}$ is exponentially stable.

(See Jacobson and Nett [5] or Curtain [2].)

Setting

$z_{s}(t):=S_{s}(t) P_{s} x_{0}+\int_{0}^{t} S_{s}(t-\tau) P_{s} B \tilde{u}(\tau) \mathrm{d} \tau$

and

$z_{u}(t):=S_{u}(t) P_{u} \tilde{x}_{0}+\int_{0}^{t} S_{u}(t-\tau) P_{u} \tilde{B} \tilde{u}(\tau) \mathrm{d} \tau$,

where $S_{u}(t):=\left.S(t)\right|_{X_{u}}$, we obtain from (3.12),

$\tilde{x}(t)=z_{u}(t)+(\lambda I-A)^{-1} z_{s}(t)$.

Since $\tilde{x}, z_{s} \in L^{2}(0, \infty ; X) \cap L^{\infty}(0, \infty ; X)$ and

$\lim _{t \rightarrow \infty} \tilde{x}(t)=\lim _{t \rightarrow \infty} z_{s}(t)=0$, the same is true for $z_{u}(t)$. Realizing that $(\lambda I-$ A) $\left.\right|_{X_{u}}$ is a bounded operator $\left(X_{u} \subset D(A)\right.$ is finite-dimensional) it follows from

$x(t)=(\lambda I-A) \tilde{x}(t)=z_{s}(t)+\left.(\lambda I-A)\right|_{X_{u}} z_{u}(t)$

that $x \in L^{2}(0, \infty ; X) \cap L^{\infty}(0, \infty ; X)$ and

$\lim _{t \rightarrow \infty} x(t)=0$.

Hence the pair $(x(t), k(t))$ satisfies (3.3)-(3.5).

In Theorem 3.4 it was required that (A4) and (A5) hold. The next two results show that either (A4) or (A5) become superfluous provided that the semigroup $S(t)$ generated by $A$ is analytic.

3.8. Theorem. If (A1)-(A4) are satisfied, $N$ is a continuously differentiable Nussbaum gain and the semigroup $S(t)$ generated by $A$ is analytic, then statement (i) of Theorem 3.4 holds true.

Proof. As in the proof of Theorem 3.4 we can show that the closed-loop system given by (2.1) and (3.1) is equivalent to the system (3.6)-(3.9). Since $B F_{\gamma}$ is an $A$-degenerate operator it follows from Zabczyk [16] that $A+B F_{\gamma}$ generates an analytic semigroup $S_{\gamma}(t)$. Now analytic semigroups satisfy the spectrum determined growth assumption and hence $S_{\gamma}(t)$ is exponentially stable by Lemma 2.5. The stability result follows from [9] as in the proof of Theorem 3.4 provided that

(i) the transfer function $H$ of (3.7) belongs to $H_{\alpha}^{\infty}$ for some $\alpha<0$, and

(ii) the function $f(t):=F_{\gamma} S_{y}(t) P_{2} x_{0}$ produced by the initial condition is in $L^{2}(0, \infty)$.

Notice that (i) and (ii) do not follow trivially because $F_{\gamma}$ is unbounded. Define

$R:=A+B F_{\gamma}, \quad D:=-(C B)^{-1} C A B(C B)^{-1}$

and

$E:=P_{2} A B(C B)^{-1}$.

It follows from Appendix II that the transfer function $H$ of (3.7) is given by

$H(s)=F_{\gamma}(s I-R)^{-1} E+D$.

Using the fact that $0 \in \rho(R)$ we obtain

$$
\begin{aligned}
H(s) & =F_{\gamma} R^{-1} R(s I-R)^{-1} E+D \\
& =F_{\gamma} R^{-1}\left(s(s I-R)^{-1}-I\right) E+D \\
& =s F_{\gamma} R^{-1}(s I-R)^{-1} E-F_{\gamma} R^{-1} E+D .
\end{aligned}
$$


Now realizing that $F_{\gamma} R^{-1}$ is a bounded operator (by the closed-graph theorem) and using that $R$ generates an exponentially stable analytic semigroup it follows that there exist $\beta<0$ and $M>0$ such that $H$ is holomorphic on $\mathbb{C}_{\beta}$ and $\left\|(s I-R)^{-1}\right\| \leq \frac{M}{|s-\beta|}$ for all $s \in \mathbb{C}_{\beta}$.

Hence $H \in H_{\alpha}^{\infty}$ for all $\alpha>\beta$, which shows that (i) holds true.

In order to prove (ii), write

$f(t)=F_{\gamma} R^{-1} R S_{\gamma}(t) P_{2} x_{0}=F_{\gamma} R^{-1} S_{\gamma}(t) R P_{2} x_{0}$

where we have used that $P_{2} x_{0} \in D(A)$ which is true because $x_{0} \in D(A)$ and im $P_{1} \subset D(A)$.

3.9. Corollary. If (A1)-(A3) and (A5) are satisfied, $N$ is a continuously differentiable Nussbaum gain and the semigroup generated by $A$ is analytic then the statements (i) and (ii) of Theorem 3.4 hold.

Proof. Define $\tilde{A}, \tilde{B}$ and $\tilde{C}$ as in the proof of Corollary 3.7 and verify that the system given by $(\tilde{A}, \tilde{B}, \tilde{C})$ fulfils (A1)-(A4). Application of Theorem 3.8 gives that for $x_{0} \in X$ the solution $(\tilde{x}(t), \tilde{k}(t))$ of the initial value problem (3.11) satisfies (3.3)-(3.5) with $x$ and $k$ replaced by $\tilde{x}$ and $\tilde{k}$. Now proceed as in the proof of Corollary 3.7.

3.10. Remark. Notice that Theorem 3.8 and Corollary 3.9 improve the result by Kobayashi [7]. They give an affirmative answer to the question raised in [7] whether the assumption that both (A4) and (A5) are satisfied can be relaxed.

\section{Appendices}

\section{Appendix $I$}

In the proof of Lemma 2.5 we have made use of the following result:

4.1. Lemma. If the operator $F_{\gamma}$ is given by (2.2) then

$$
\overline{\mathbf{C}}_{0} \cap \rho\left(A+B F_{\gamma}\right) \neq \varnothing \text {. }
$$

Proof. Set $G_{\gamma}(s):=F_{\gamma}(s I-A)^{-1} B$. Since $A$ generates a strongly continuous semigroup there exists $\alpha \in[0, \infty)$ such that $\mathbb{C}_{\alpha} \subset \rho(A)$. Hence $G_{\gamma}(s)$ is well defined for all $s \in \mathbb{C}_{\alpha}$.

Step 1: We claim that $s \in \rho\left(A+B F_{\gamma}\right)$ if $s \in \mathbb{C}_{\alpha}$ and $G_{\gamma}(s) \neq 1$. Notice that for $s \in \mathbb{C}_{\alpha}$,

$$
I=\left(s I-A-B F_{\gamma}\right)(s I-A)^{-1}+B F_{\gamma}(s I-A)^{-1}
$$

so that

$$
B\left(1-G_{\gamma}(s)\right)=\left(s I-A-B F_{\gamma}\right)(s I-A)^{-1} B .
$$

For $s \in \mathbb{C}_{\alpha}$ satisfying $G_{\gamma}(s) \neq 1$ we obtain

$$
B=\left(s I-A-B F_{\gamma}\right)(s I-A)^{-1} B\left(1-G_{\gamma}(s)\right)^{-1} \text {. }
$$

Substituting (4.3) into (4.1) gives

$$
\begin{aligned}
I= & \left(s I-A-B F_{\gamma}\right)(s I-A)^{-1} \\
& \cdot\left[I+B\left(1-G_{\gamma}(s)\right)^{-1} F_{\gamma}(s I-A)^{-1}\right] .
\end{aligned}
$$

We obtain from the definition of $F_{\gamma}$ that the operator

$$
\begin{aligned}
H_{\gamma}(s):= & (s I-A)^{-1} \\
& \cdot\left[I+B\left(1-G_{\gamma}(s)\right)^{-1} F_{\gamma}(s I-A)^{-1}\right]
\end{aligned}
$$

is bounded. Equation (4.4) shows that $H_{\gamma}(s)$ is a right inverse of $s I-A-B F_{\gamma}$. The claim now follows since it is not difficult to show that $H_{\gamma}(s)$ is a left inverse of $s I-A-B F_{\gamma}$ as well.

Step 2: It remains to show that there exists $\xi \in \mathbb{C}_{\alpha}$ satisfying $G_{\gamma}(\xi) \neq 1$. We will prove that

$$
\lim _{\lambda \rightarrow \infty} G_{\gamma}(\lambda)=0
$$

where $\lambda$ is a real variable. Since $A$ generates a strongly continuous semigroup there exist real numbers $M$ and $\beta$ such that

$\left\|(\lambda I-A)^{-1}\right\| \leq \frac{M}{\lambda-\beta}$ for all $\lambda>\beta$.

In order to prove that (4.5) holds true it is sufficient to show

$\lim _{\lambda \rightarrow \infty}\left\|A(\lambda I-A)^{-1} B\right\|=0$. 
Notice that

$$
\begin{aligned}
\left\|A(\lambda I-A)^{-1}\right\| & =\left\|\lambda(\lambda I-A)^{-1}-I\right\| \\
& \leq 1+\frac{M \lambda}{\lambda-\beta} \quad \forall \lambda>\min (0, \beta) .
\end{aligned}
$$

Thus there exists $\lambda_{0}>\max (0, \beta)$ such that

$\left\|A(\lambda I-A)^{-1}\right\| \leq 1+2 M \quad \forall \lambda>\lambda_{0}$.

Let $\varepsilon>0$ be given. Set $x:=B(1)$ and choose $z \in$ $D(A)$ satisfying

$\|x-z\| \leq \frac{\varepsilon}{2(1+2 M)}$.

Moreover let $\lambda_{1} \geq \lambda_{0}$ be such that

$\frac{M}{\lambda-\beta}\|A z\| \leq \frac{1}{2} \varepsilon \quad \forall \lambda>\lambda_{1}$.

Then it follows from (4.6) and (4.8)-(4.10),

$$
\begin{aligned}
\left\|A(\lambda I-A)^{-1} B\right\|= & \left\|A(\lambda I-A)^{-1} x\right\| \\
\leq & \left\|A(\lambda I-A)^{-1}\right\|\|x-z\| \\
& +\left\|(\lambda I-A)^{-1}\right\|\|A z\| \\
\leq & \varepsilon \quad \forall \lambda>\lambda_{1},
\end{aligned}
$$

which proves (4.7)

\section{Appendix $I I$}

Consider the system

$$
\begin{aligned}
& \dot{x}(t)=A x(t)+B u(t), \quad x(0)=x_{0}, \\
& y(t)=C x(t), \quad t \geq 0,
\end{aligned}
$$

where $A$ generates a strongly continuous semigroup $S(t)$ on a Banach space $X, B \in \mathscr{L}(\mathbb{R}, X)$ and $C: D(C) \rightarrow \mathbb{R}$ is an $A$-bounded linear operator. If $x_{0} \in D(A)$ and $u \in C^{\mathbf{l}}(0, \infty ; \mathbb{R})$ there exists a unique classical solution $x(t) \in D(A)(\forall t \geq$ 0 ) and hence the output $y$ is well defined.

In the following let $\lambda$ denote the exponential growth constant of $S(t)$. As usual the Laplace transformation is denoted by the superscript .

4.2. Proposition. Suppose $x_{0}=0$ and let $u \in$ $C^{1}(0, \infty ; \mathbb{R})$ be Laplace transformable such that $\hat{u}(s)$ exists on $\mathbb{C}_{\alpha}$ for some $\alpha \in \mathbb{R}$. Then the Laplace transform $\hat{y}(s)$ of the output of (4.11) exists for all $s \in \mathbb{C}$ satisfying $\operatorname{Re}(s)>\max (\alpha, \lambda)$ and is given by

$\hat{y}(s)=C(s I-A)^{-1} B \hat{u}(s)$.
Moreover the expression $C(s I-A)^{-1} B$ is analytic in $\mathbb{C}_{\lambda}$.

4.3. Remark. The above proposition says that there exists a transfer function for the system (4.11) and that it is given by $C(s I-A)^{-1} B$. This seems like a trivial fact. However, since $C$ is unbounded, we have to prove that $C$ can be taken out of the Laplace integral.

Proof of Proposition 4.2. W.l.o.g. we may assume that $\lambda<0$ and hence $A^{-1} \in \mathscr{L}(X, X)$. It is well known from semigroup theory that

$$
\left.\frac{\mathrm{d}}{\mathrm{d} \tau}\left(A^{-1} T(\tau) B\right)\right|_{\tau=t}=T(t) B .
$$

Using (4.12), the variation-of-constants formula and partial integration we obtain

$$
\begin{aligned}
x(t)= & -\int_{0}^{t} \mathrm{~d}_{\tau}\left(A^{-1} T(t-\tau) B\right) u(\tau) \mathrm{d} \tau \\
= & \int_{0}^{t} A^{-1} T(t-\tau) B u^{\prime}(\tau) \mathrm{d} \tau \\
& -A^{-1} B u(t)+A^{-1} T(t) B u(0) .
\end{aligned}
$$

Applying $C$ to both sides of the equation, using the fact that $C A^{-1}$ is bounded and taking Laplace transforms gives

$$
\begin{aligned}
\hat{y}(s)= & C A^{-1}(s I-A)^{-1} B(s \hat{u}(s)-u(0)) \\
& -C A^{-1} B \hat{u}(s)+C A^{-1}(s I-A)^{-1} B u(0) \\
= & C A^{-1}\left\{s(s I-A)^{-1}-I\right\} B \hat{u}(s) \\
= & C A^{-1}\left\{A(s I-A)^{-1}\right\} B \hat{u}(s) \\
= & C(s I-A)^{-1} B \hat{u}(s) .
\end{aligned}
$$

It is clear that the above equations hold for all $s \in \mathbb{C}$ satisfying $\operatorname{Re}(s)>\max (\alpha, \lambda)$. Moreover it follows from the identity

$C(s I-A)^{-1} B=C A^{-1}\left\{s(s I-A)^{-1}-I\right\} B$ that $C(s I-A)^{-1} B$ is analytic in $\mathbb{C}_{\lambda}$.

\section{References}

[1] R.F. Curtain, Invariance concepts in infinite dimensions, SIAM J. Control Optim. 24 (1986) 1009-1031.

[2] R.F. Curtain, Equivalence of input-output stability and exponential stability for infinite-dimensional systems, Math. Systems Theory 21 (1988) 14-48. 
[3] M. Dahleh, Generalization of Tychonov's theorem with applications to adaptive control of SISO delay systems, Systems Control Lett. 13 (1989) 421-427.

[4] M. Dahleh and W.E. Hopkins, Jr., Adaptive stabilization of single-input single-output delay systems, IEEE Trans. Automat. Control 31 (1986) 577-579.

[5] C.A. Jacobson and C.N. Nett, Linear state-space systems in infinite-dimensional space: the role and characterization of joint stabilizability/detectability, IEEE Trans. Automat. Control 33 (1988) 541-549.

[6] T. Kato, Perturbation Theory for Linear Operators (Springer, Berlin, 2nd ed., 1976).

[7] T. Kobayashi, Global adaptive stabilization of infinite-dimensional systems, Systems Control Lett. 9 (1987) 215 223.

[8] H. Logemann, Adaptive exponential stabilization for a class of nonlinear retarded processes, Math. Control Signals Systems 3 (1990) 255-269.

[9] H. Logemann and D.H. Owens, Input-output theory of high-gain adaptive stabilization of infinite-dimensional systems with non-linearities, Internat. J. Adaptive Control and Signal Process. 2 (1988) 193-216.

[10] R.H. Martin, Jr., Nonlinear Operators and Differential Equations in Banach Spaces (J. Wiley, New York, 1976).
[11] S.A. Nefedov and F.A. Sholokhovich, A criterion for the stabilizability of dynamical systems with finite-dimensional input, Differentsial'nye Urauneniya 22 (1986) 163166.

[12] R.D. Nussbaum, Some remarks on a conjecture in parameter adaptive control, Systems Control Lett. 3 (1983) 243 246.

[13] A. Pazy, Semigroups of Linear Operators and Applications to Partial Differential Equations (Springer, New York, 1983).

[14] I. Segal, Non-linear semigroups, Ann. of Math. 78 (1963) 339-364.

[15] J.C. Willems and C.I. Byrnes, Global adaptive stabilization in the absence of information on the sign of the high-frequency gain, in: Proc. INRIA Conf. on Analysis and Optimization of Systems, Lecture Notes in Control and Information Sciences, No. 62 (Springer, New York, 1984) 49-57.

[16] J. Zabczyk, On decomposition of generators, SIAM J. Control Optim. 16 (1978) 523-534.

[17] H.J. Zwart, Equivalence between open-loop and closedloop invariance for infinite-dimensional systems: a frequency-domain approach, SIAM J. Control Optim. 26 (1988) 1175-1199. 